\title{
Economics of climate change and risk of disasters in Asia-Pacific region
}

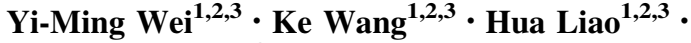 \\ Hirokazu Tatano ${ }^{4}$
}

Published online: 7 October 2016

(C) Springer Science+Business Media Dordrecht 2016

Asia-Pacific region is one of the most dynamic regions in the world. During the latest three decades, the economic progress of nations in this region has been remarkable, which is characterized by rapid growth in economic prosperity, especially East Asia countries. However, the current huge amount and unsustainable energy consumption as well as rapid increase in carbon emission and pollutant emission in Asia-Pacific region has already constrained the continuous development of some nations. Furthermore, global climate change also begun to threaten the prospect of prosperity in East Asia countries and other emerging nations. The characteristics of geography, population density, distribution of industry and natural resource make this region one of the most vulnerable regions to climate change and related natural hazards. Researches on development economics, energy economics as well as economics of climate change and natural hazards covering AsiaPacific region-such as assessment of climate change and natural hazard impacts, evaluation of adaptation and mitigation strategies and policies, modeling energy conservation and carbon emission reduction scenarios, and analyzing the relationship among economic growth, energy and resources consumption, carbon and pollutants emissions, national welfare and social progress - are very important and obviously useful in climate change adaptation and mitigation planning. Therefore, this special issue aims to promote and publish researches on economics of climate change and risk of disasters in Asia-Pacific region and around the world for a better understanding of existing knowledge and research gaps.

Yi-Ming Wei

wei@bit.edu.cn

1 Center for Energy and Environmental Policy Research \& School of Management and Economics, Beijing Institute of Technology, Beijing, China

2 Collaborative Innovation Center of Electric Vehicles in Beijing, Beijing, China

3 Sustainable Development Research Institute for Economy and Society of Beijing, Beijing, China

4 Disaster Prevention Research Institute, Kyoto University, Kyoto, Japan 
In this special issue, we collected 23 contributions which showed variety of research topics of interest in economics of climate change and risk of disasters in Asia-Pacific region.

I. Noy provided a new measurements of impacts of natural disasters in the Pacific Island Countries. In this paper, the burden of disasters on these countries was tabulated and measured in three steps. Firstly, the data found in the two global public datasets on disaster impacts were aggregated and compared. Secondly, a new index that aggregates disparate disaster impacts was developed and calculated for each Pacific Island Country. Thirdly, the burden of disasters on the Pacific Island Countries was compared with that on the Caribbean Island Countries. The results of this paper showed that the most commonly used dataset, EMDAT, greatly underestimates the burden of disasters on the Pacific Islands, and the burden of disasters is significantly more acute in the Pacific.

J. A. Lassa, A. Y. H. Lai and T. Goh contributed a research on an observation and projection of impacts of climate extremes on food production in ASEAN. They pointed out that, since climate hazards may differ across geographical regions, the impact of climate extremes on food production will affect marginal farmers, fishers and poor urban consumers disproportionately, as they have limited capacities to adapt to and recover from extreme weather events. However, there was still limited study on this subject matter. This paper presented some findings from research on observed and projected loss and damage inflicted by climate extremes on agricultural crops in Southeast Asia.

X. Jiang, N. Mori, H. Tatano, L. Yang and Y. Shibutani provided an estimation of property loss and business interruption loss under scenarios of storm surge inundation to explore the economic impact of climate change on Ise Bay of Japan, in which the scenarios-based analyses were conducted with respect to Typhoon Vera, which caused the most severe storm surge in the recorded history of Japan in 1959. By comparing the property loss and business interruption loss caused by different hazard scenarios, the effect of different seawalls was evaluated and the economic impact of future climate change was estimated in this paper. Their results indicated that although the current seawall would considerably reduce the scale of losses, climate change would cause Ise Bay to experience more serious storm surge inundation, and result in a significant increase in economic losses owing to a combination of climate change and the worst track scenario.

C. J. Yang and H. Huang contributed a paper named Mapping Tropical Forest Vegetation from LANDSAT TM images Based on Fusion of Knowledge and Geo-data. In this paper, a rule-based model to identify different forest types in Xishuangbanna of China, using two temporal Landsat TM images and geo-data such as DEM, rainfall and temperature, was developed. The results of this model showed that it is useful and effective in tropical forest vegetation mapping, which can effectively integrate multi-knowledge and multi-resource data to identify the tropical forest vegetation types with higher accuracy.

X. H. Yang, B. Y. Sun, J. Zhang, M. S. Li, J. He, Y. M. Wei and Y. Q. Li provided a hierarchy evaluation of water resources vulnerability to climate change in Beijing of China. In this paper, a multi-functional hierarchy indicator system for the performance evaluation of water resources vulnerability (WRV) under climate change was developed, and an evaluation model named analytic hierarchy process combining set pair analysis (AHPSPA) model was established for assessing WRV. This model was used to assess water resource vulnerability in Beijing with 26 indexes under eight kinds of future climate change scenarios. Their results showed that the WRV of Beijing was in the middle vulnerability and water resources change and water use level factors played more important role in the evaluation system of water resource vulnerability in Beijing. 
$\mathrm{X}$. Yu discussed the central-local conflicts in China's environmental policy implementation. She pointed out in this paper that like many other environmental policies, forestry policy has not been effectively implemented in China, mainly due to the fragmentation nature in China's environmental governance institution. With insufficient motivation, local governments tend to minimize their efforts in planning, monitoring and supporting reforestation activities, which poses great challenges to the sustainability of the reforestation benefits. This paper raised three innovative strategies to solve the financial dilemma that leads to the effort minimization phenomenon, with both the advantages and disadvantages for each strategy critically discussed. This paper also recommended ways by which the central government could improve design of reforestation policies, or other large-scale ecological programs, which involve local governments as a key liaison.

J. Hou, J. Lv, X. Chen and S. Yu provided an evaluation and spatial characteristics analysis of China's regional social vulnerability to geological disasters. In this paper, the social vulnerability index of geological disasters was calculated through a super-efficiency data envelopment analysis model. In addition, the global and local autocorrelation tests for social vulnerability to geological disasters in each province in China were carried out which indicated the characteristics of its spatial distribution pattern. The results showed that China's social vulnerability to geological disasters is relatively high; the social vulnerability index of geological disasters in China is directly related to the regional exposure degree and reaction and recovery ability and among which the reaction and recovery ability has great effects on the social vulnerability index.

B. Tang, Y. Hu, H. Li, D. Yang and J. Liu provided an estimation of comprehensive carrying capacity of Beijing-Tianjin-Hebei region of China. In this paper, based on a statespace method and a component analysis, a comprehensive evaluation system of carrying capacity was established for the Beijing-Tianjin-Hebei region from four aspects: economy, environment, ecology and energy. Their evaluation results showed that the comprehensive carrying capacity in this region gradually rose in recent years and the economic carrying capacity played an important role. Ecological and environmental carrying capacity gradually enhanced but was still affected by water shortages. The energy carrying capacity was low restricting the sustainable development of this region.

P. Gong, B. Tang, Y. Xiao, G. Lin and J. Liu contributed a paper on exploring the embodied carbon in China's export structure. Their calculation showed that the total carbon emission of export experienced an increase before 2007 and then a decrease till 2010. The ratio of export embodied carbon accounting for the total carbon emission in China also increased from $31 \%$ in 2002 to $52 \%$ in 2007 and then declined to $40 \%$ in 2010. They found that the products with strong international competitiveness in China belong to high-carbon-emission categories, which was the major reason for high carbon emission in China export.

M. Ikram, Z. Yan, Y. Liu and D. Wu provided an assessment of the possible impacts of temperature change on air quality and public health in Beijing, China. In their paper, the ordinary least-squares method was utilized to examine the impact of temperature fluctuations on air quality index (AQI) and respiratory health outcomes (RHOs) during 2008 and 2012 in Beijing. Their results showed that a drop of more than $3{ }^{\circ} \mathrm{C}$ would result in the increased impact on AQI and RHO in the heating period, while a raise of more than $3{ }^{\circ} \mathrm{C}$ results in the similar increased impact on AQI both in the whole study period and the heating period. Furthermore, an increase in temperature also results in the increased influence of health risk on females during the heating period.

Z. Zhu, H. Zhang, G. Tao and F. Yu analyzed the potential effects of natural gas pricing reform on China's price level and total output in their paper based on an input-output model. Their results showed that the pricing reform had relatively higher impact on the 
products' price of the sectors that have large gas consumption; effects of gas pricing reform on urban and rural residents were dissymmetrical, with larger effects on urban residents; however, the pricing reform has relatively small impacts on various price indices and total output levels in China.

L. He, C. Hu, D. Zhao, H. Lu, X. Fu and Y. Li contributed a literature review-based investigation on proposing a theoretical framework of carbon efficient supply chain management. This paper generated concepts with a formal framework of potential and values theoretical issues in the emerging field of carbon efficient supply chain management which combines carbon emission regulations with mature operation management to enrich the theory of supply chain management.

W. Y. Liu and Q. Wang established several theoretical models of supply and demand for carbon trading and proposed conditions for optimal trading prices and periods in their paper. The carbon market in Taiwan was used to verify the validity of their models. Four trading scenarios to determine optimal trading prices and time periods were compared in their paper, and one of their key findings was that the government should design separate carbon programs and trading mechanisms for different types of private landowners so as to strengthen the incentives for participating in the carbon trading program.

M. Song, G. Zhang, K. Fang and J. Zhang provided an evaluation of China's regional operational and environmental performance. In this paper, a non-radial data envelopment analysis model under natural and managerial disposability was utilized to measure the unified efficiency of 30 administrative regions in China.

L. Chen and Q. Duan evaluated the driving factors impacting China's carbon emissions during 2001 and 2010. The results of this paper showed that changes in scale effect and changes in inputs were the major driving factors for carbon emissions growth, while technical efficiency, technological progress and change in output structure inhibited carbon emissions growth.

J. Wu, J. F. Chu and L. Liang proposed a model for target setting and allocation of carbon emissions abatement and then applied this model to 20 APEC economies. In this paper, they pointed out that the traditional carbon emissions abatement allocation methods generally set efficient targets for the decision-making units using the farthest targets, which neglects the decision-making units' unwillingness to maximize (or minimize) some of their inputs (or outputs). To surmount this deficiency, they incorporated the data envelopment analysis and its closest target technique into the carbon emissions abatement allocation problem. Their approach provided more flexibility when setting new input and output targets for the decision-making units in the allocation. The proposed approaches were further applied for carbon emissions abatement target setting and allocation for 20 AsiaPacific Economic Cooperation economies.

B. Han, D. Wang, W. Ding and L. Han provided an empirical analysis on the effect of information and communication technology on energy consumption in China. The major findings of this paper showed that the effect of information and communication technology on energy consumption can be represented by a U-shaped curve. The influence of population, income, industrialization level, service sector development, energy price, energy consumption structure and non-information and communication technology capital service on energy consumption was also examined in this paper.

Y. Chen, B. Han and W. Liu provided an empirical analysis on the effect of green technology innovation on energy intensity in China for its 29 sample provinces which were divided into four groups: conventional group, gradational group, contemporary group and low-carbon group. Their testing results indicated that there was a negative, long-run, cointegrated relationship between energy intensity and green technology innovation, and 
the green technology innovation directly affects energy intensity through a feedback system for the gradational and contemporary groups.

Y. F. Yao and Q. M. Liang contribute a computable general equilibrium-based analysis of carbon allowance allocation in China. In this paper, the China Energy and Environmental Policy Analysis (CEEPA) model was applied to assess how the carbon allowance should be appropriately allocated. The simulation results of this model showed that, while the impacts on China's economic development varied according to how allowances were allocated, the negative impacts could not be completely mitigated, which were between -0.5 and $-0.1 \%$ when $5 \%$ of carbon emissions were reduced. One important suggestion was that, if citizens' welfare and quality of life was prioritized in the allocation, auctioning the allowance and transferring the revenue to households in proportion to their occupation would be the most effective approach; in this case, the negative impacts on rural households' disposable incomes and welfare would be reduced, and the income gap between rural and urban households would be narrowed.

Q. Wang, Q. M. Liang, B. Wang and F. X. Zhong contributed an analysis on the relationship between household expenditure and carbon emissions among different income groups of urban and rural households in China. Through utilizing China's 2007 Social Accounting Matrix, they examined the direct and indirect carbon emissions caused by household demand. The major result indicated that the income gap and the difference in consumption propensity were the primary causes of the significant differences in emission levels. Another interesting result was that the rural higher-income groups spend a larger share of their income on those carbon-intensive goods, thus making their consumption patterns more carbon-intensive, while for the urban, the consumption patterns of lower income groups are more carbon-intensive.

L. Yang, Y. Kajitani, H. Tatano and X. Jiang proposed a probabilistic methodology for estimating the business interruption loss of industrial sectors caused by flood disasters as an extension of current methodology. In this paper, the functional forms and parameters were selected and calibrated based on survey data obtained from businesses located in the inundated area which was affected by the 2000 Tokai Heavy Rain. This heavy rain was a rare event that hit a densely populated and industrialized area. Explanatory variables like inundation depth, distinct vulnerability, resilience characteristics of each sector, and the accuracy of fit of the model were analyzed in this paper. The authors pointed out that the function obtained and the parameters estimated could be further utilized as benchmarks in estimating the probabilistic distribution of business interruption losses, especially in the case of urban flood disasters.

We are glad to become guest editors for the special issue of economics of climate change and risk of disasters in Asia-Pacific region. We do hope this special issue will be of interest to academic and industrial communities, as well as policy makers for helping them to understand the status and complexity of climate change and risk of disasters in AsiaPacific region from the economics perspectives.

In closing, we thank the anonymous referees for their efforts during the review process. We gratefully acknowledge Professor Tad S. Murty, Editor of Natural Hazards, Petra van Steenbergen, Executive Editor of Springer, Stefan van Dijl, Editor of Springer, and Fazil Rafiq, Journals Editorial Office Assistant, for supporting the special issue and offering their expert guidance and efforts to make this issue published.

We acknowledge the financial support from the National Natural Science Foundation of China under Grant Nos. 71471018, 71521002, 71673026, the Social Science Foundation of Beijing under Grant No. 16JDGLB013, and the National Key R\&D Program under Grant No. 2016YFA0602603. 